\title{
Apoptosis in nonalcoholic steatohepatitis with normal aminotransferase values: zooming in on
}

\section{cytokeratin 18 fragments}

A letter in response to: Canbakan B, Senturk H, Canbakan M et al.: Is alanine aminotransferase level a surrogate biomarker of hepatic apoptosis in nonalcoholic fatty liver disease? Biomarkers Med. 4(2), 205-214 (2010).

\section{KEYWORDS: alanine aminotransferase $\approx$ apoptosis $\approx$ nonalcoholic fatty liver disease}

Recent years have witnessed an increasing interest in the relationship between alanine aminotransferase (ALT) and nonalcoholic fatty liver disease (NAFLD) [1]. Traditionally, increased ALT concentrations are believed to be a consequence of liver injury in NAFLD and are, thus, widely used as a surrogate marker of this condition [2]. However, studies assessing the specificity and sensitivity of ALT as a marker of NAFLD have been limited and efforts to identify reliable cut-off values for this enzyme, in order to facilitate the identification of subjects with NAFLD, have generated conflicting results [3]. Another issue inherent to the use of ALT as a biomarker of NAFLD is the possible confounding factors that might cause ALT elevation [4]. For example, small amounts of alcohol consumption, as well as hepatotoxic medications, may act as confounding factors in the association between increased ALT concentrations and NAFLD. These caveats notwithstanding, ALT can be considered an acceptable marker for NAFLD in epidemiological studies. By contrast, caution should exercised regarding the use of ALT for diagnostic and monitoring purposes in the clinical care of NAFLD patients [5]. Another problem with the use of ALT as a diagnostic or monitoring biomarker of NAFLD is the existence of two isoforms of this enzyme, termed ALT1 and -2 [6]. Jadhao and colleagues have previously demonstrated that murine ALT2 gene expression is induced twofold in fatty livers of obese mice [7]. By contrast, the expression of murine ALT1 was unchanged. This raises a question regarding which ALT form is actually measured in serum in the settings of standard clinical chemistry.

It is now widely accepted that the full histological spectrum of NAFLD may be found in patients with normal ALT. Francanzani and coworkers have convincingly demonstrated that
NAFLD patients with normal ALT are at risk of progressive and severe hepatic disease, as well as of extrahepatic manifestations [8]. In the light of their results, the authors concluded that this evidence could make liver biopsy mandatory in the majority of cases, unless currently unavailable sensitive and specific noninvasive tests prove their efficacy [8]. Unfortunately, liver biopsy has a number of limitations, including considerable sampling variability, patient discomfort, potential morbidity (bleeding and organ perforation) and costs $[9,10]$. Of note, serious complications have been demonstrated to occur in $0.3 \%$ of liver biopsies, with $0.01 \%$ being fatal [9].

\section{Cytokeratin 18 fragments, NAFLD \& normal ALT}

In the April 2010 edition of Biomarkers in Medicine, Canbakan and colleagues reported the results of their study, exploring the significance of ALT elevation in patients with NAFLD [11]. The authors demonstrate that ALT elevation was not associated with advanced necroinflammatory grade and fibrosis. Interestingly, ALT values did not correlate with the parameters of apoptosis and oxidative stress. In keeping with the results of Fracanzani and colleagues, the authors concluded that only liver biopsy can be used for accurately determining the histological severity and prognosis of liver damage in the setting of metabolic liver diseases [8]. Of note, the authors demonstrated that there was no association between ALT and the immunohistochemical expression of caspase 3 and 8 in the liver [11].

Growing evidence has recently suggested that dysregulation of the apoptotic pathway in hepatocytes plays an important role in a wide range of chronic liver disorders [12]. A neoepitope in cytokeratin (CK) 18 becomes available at an early caspase cleavage event during hepatocyte apoptosis,

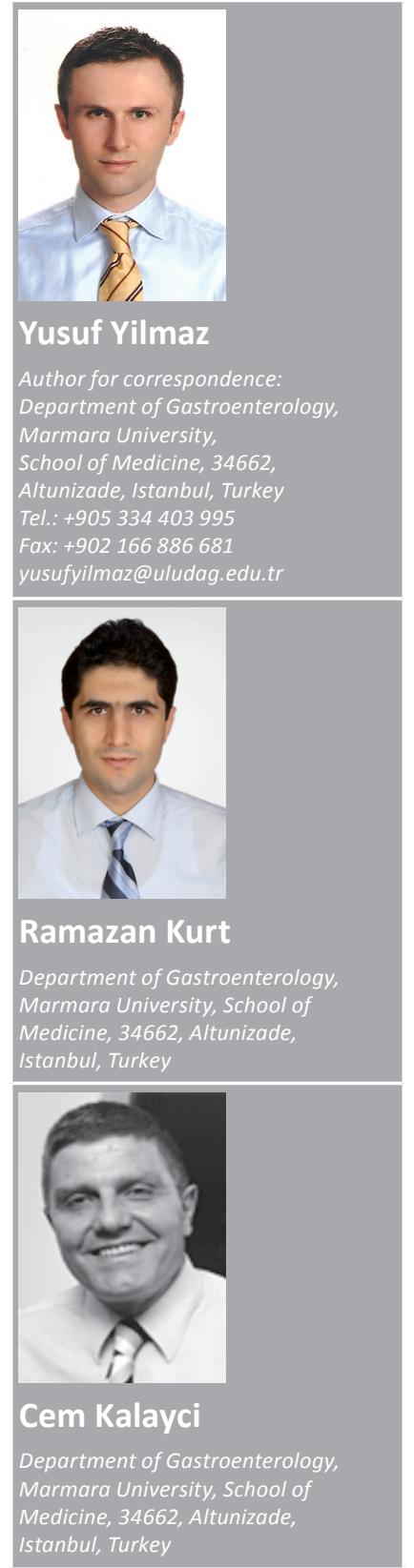

future medicine $^{\text {pat }}$ fsg 
and is not detectable in vital or necrotic cells [13]. A monoclonal antibody, M30, specifically recognizes the fragment of CK18, which is cleaved at Asp396 (M30 antigen). An M30-based sandwich ELISA assay determines the circulating levels of M30 antigen and may serve as a surrogate serum biomarker of hepatocyte apoptosis [14,15]. CK18 is cleaved, primarily by caspase 9 , to generate this M30 neoepitope, but also by caspase 3 and 7 [16] The assessment of serum M30 antigen holds great promise for assessing the degree of liver injury in several chronic liver diseases, including NAFLD [13]. Importantly, we have recently demonstrated that serum M30 levels may be associated with the presence of more severe inflammatory changes (nonalcoholic steatohepatitis [NASH]) among NAFLD cases with normal ALT levels [17]. We have enrolled 18 patients with biopsy-confirmed NAFLD and normal ALT levels (<40 U/l). Compared with patients with NAFLD, but not NASH $(\mathrm{n}=12)$, mean serum M30 levels were significantly raised in the six patients with normal ALT and NASH levels $(198.2 \pm 37.2 \mathrm{U} / \mathrm{l}$ vs $80.7 \pm 19.2 \mathrm{U} / \mathrm{l}, \mathrm{p}<0.01)$. In multivariable analysis, M30 levels were significantly and independently associated with the presence of NASH in patients with NAFLD and normal ALT levels [17]. It is noteworthy that, although activated caspase 3 immunohistochemistry is recommended for the detection and quantification of apoptosis in tissue sections [18], M30 levels are mainly produced by caspase 9 during apoptotic cell death of hepatocytes [16]. Therefore, it is possible that serum M30 levels [13] and liver expression of caspase 3 and 8 [11] would actually capture different aspects of hepatocyte apoptosis occurring in the setting of NAFLD. However, it should be noted that the diagnostic performance of serum M30 levels alone may be suboptimal [19]. In addition, the diagnostic value of M30 levels for predicting histological NASH and the severity of the disease may be confounded by the amount of fibrosis [20]. Another potential caveat of only using serum M30 concentrations is the lack of specificity for NASH as levels of this molecule may be altered in other disease conditions, such as cholecystitis [21], cancers [22] and coronary artery disease [23]. One potential strategy to overcome this issue may lie in the measurement of different molecular forms of CK18 released from dead cells. While apoptosis is associated with the release of M30 antigen, necrosis will result in release of uncleaved CK18 (M65 antigen) [24]. It is worth noting that there is also evidence suggesting that levels of M65 antigen may be of value for determining the severity of liver injury in the spectrum of NAFLD $[15,19]$.

\section{Conclusion}

Up until now, there has been a paucity of studies devoted to finding a reliable biomarker of severe liver disease in NAFLD patients with normal ALT. Clearly, a liver biopsy cannot be performed in all patients with NAFLD $[10,25,26]$. The assessment of serum levels of cleaved and intact CK18 (M30 and M65 antigen, respectively) appears to hold promise as an alternative to liver biopsy as a reliable way of diagnosing NASH and monitoring disease progression in patients with NAFLD and normal ALT [11]. Future validation studies and developments in this field can make this approach ideal for screening and monitoring purposes.

\section{Financial \& competing interests disclosure}

The authors have no relevant affiliations or financial involvement with any organization or entity with a financial interest in or financial conflict with the subject matter or materials discussed in the manuscript. This includes employment, consultancies, honoraria, stock ownership or options, expert testimony, grants or patents received or pending, or royalties.

No writing assistance was utilized in the production of this manuscript.

7 Jadhao SB, Yang RZ, Lin Q et al.: Murine alanine aminotransferase: cDNA cloning, functional expression, and differential gene regulation in mouse fatty liver. Hepatology 39, 1297-1302 (2004). (2007).

2 Schindhelm RK, Diamant M, Dekker JM et al: : Alanine aminotransferase as a marker of non-alcoholic fatty liver disease in relation to Type 2 diabetes mellitus and cardiovascular disease. Diabetes Metab. Res. Rev. 22, 437-443 (2006).

3 Lee JK, Shim JH, Lee HC et al.: Estimation of the healthy upper limits for serum alanine aminotransferase in
4 Calvaruso V, Craxì A: Implication of normal liver enzymes in liver disease. J. Viral Hepat. 16, 529-536 (2009).

Asian populations with normal liver histology. Hepatology 51, 1577-1583 (2010).

5 Prati D, Taioli E, Zanella A et al.: Updated definitions of healthy ranges for serum alanine aminotransferase levels. Ann. Intern. Med. 137, 1-10 (2002).

6 Yang RZ, Park S, Reagan WJ et al.: Alanine aminotransferase isoenzymes: molecular cloning and quantitative analysis of tissue expression in rats and serum elevation in liver toxicity. Hepatology 49, 598-607 (2009).
Fracanzani AL, Valenti L, Bugianesi E et al:: Risk of severe liver disease in nonalcoholic fatty liver disease with normal aminotransferase levels: a role for insulin resistance and diabetes. Hepatology 48, 792-798 (2008).

9 Wieckowska A, Feldstein AE: Diagnosis of nonalcoholic fatty liver disease: invasive versus noninvasive. Semin. Liver Dis. 28, 386-395 (2008). 
10 Hübscher SG: Role of liver biopsy in the assessment of non-alcoholic fatty liver disease. Eur. J. Gastroenterol. Hepatol. 16, 1107-1115 (2004).

11 Canbakan B, Senturk H, Canbakan $M$ et al: Is alanine aminotransferase level a surrogate biomarker of hepatic apoptosis in nonalcoholic fatty liver disease? Biomark. Med. 4(2), 205-214 (2010).

12 Feldstein AE, Canbay A, Angulo P et al: Hepatocyte apoptosis and fas expression are prominent features of human nonalcoholic steatohepatitis. Gastroenterology 125, 437-443 (2003).

13 Yilmaz Y: Systematic review: caspase-cleaved fragments of cytokeratin 18 - the promises and challenges of a biomarker for chronic liver disease. Aliment. Pharmacol. Ther. 30, 1103-1109 (2009).

14 Yilmaz Y, Kedrah AE, Ozdogan O: Cytokeratin-18 fragments and biomarkers of the metabolic syndrome in nonalcoholic steatohepatitis. World J. Gastroenterol. 15, 4387-4391 (2009).

15 Yilmaz Y, Dolar E, Ulukaya E et al:: Soluble forms of extracellular cytokeratin 18 may differentiate simple steatosis from nonalcoholic steatohepatitis. World J. Gastroenterol. 14, 837-844 (2007).
16 Vos MB, Barve S, Joshi-Barve S et al:: Cytokeratin 18, a marker of cell death, is increased in children with suspected nonalcoholic fatty liver disease. J. Pediatr. Gastroenterol. Nutr. 47, 481-485 (2008).

17 Yilmaz Y, Ulukaya E, Dolar E: Serum M30 levels: a potential biomarker of severe liver disease in nonalcoholic fatty liver disease and normal aminotransferase levels. Hepatology 49, 697 (2009).

18 Duan WR, Garner DS, Williams SD et al.: Comparison of immunohistochemistry for activated caspase- 3 and cleaved cytokeratin 18 with the TUNEL method for quantification of apoptosis in histological sections of PC-3 subcutaneous xenografts. J. Pathol. 199, 221-228 (2003).

19 Younossi ZM, Jarrar M, Nugent C et al.: A novel diagnostic biomarker panel for obesity-related nonalcoholic steatohepatitis (NASH). Obes. Surg. 18, 1430-1437 (2008).

20 Feldstein AE, Wieckowska A, Lopez AR et al.: Cytokeratin-18 fragment levels as noninvasive biomarkers for nonalcoholic steatohepatitis: a multicenter validation study. Hepatology 50, 1072-1078 (2009).
21 Simopoulos C, Tsaroucha AK, Asimakopoulos B et al.: Total and caspasecleaved cytokeratin 18 in chronic cholecystitis: a prospective study. BMC Gastroenterol. 8, 14 (2008).

22 Barak V, Goike H, Panaretakis KW, Einarsson R: Clinical utility of cytokeratins as tumor markers. Clin. Biochem. 37, 529-540 (2004).

23 Senturk T, Aydinlar A, Yilmaz Y et al.: Serial changes in circulating M30 antigen, a biomarker of apoptosis, in patients with acute coronary syndromes: relationship with the severity of coronary artery disease. Coron. Artery Dis. 20, 494-498 (2009).

24 de Haas EC, di Pietro A, Simpson KL et al.: Clinical evaluation of M30 and M65 ELISA cell death assays as circulating biomarkers in a drug-sensitive tumor, testicular cancer. Neoplasia 10, 1041-1048 (2008).

25 Emanuele E: Is biopsy always necessary? Toward a clinico-laboratory approach for diagnosing nonalcoholic steatohepatitis in obesity. Hepatology 48, 2086-2087 (2008).

26 Yilmaz Y, Ulukaya E, Dolar E: A "biomarker biopsy" for the diagnosis of NASH: promises from CK-18 fragments. Obes. Surg. 18, 1507-1508 (2008). 